\title{
Online Games and Family Ties: Influences of Social Networking Game on Family Relationship
}

\author{
Jing Wen ${ }^{1,2}$, Yong Ming Kow ${ }^{1}$, and Yunan Chen ${ }^{1}$ \\ ${ }^{1}$ Department of Informatics \\ University of California, Irvine \\ Irvine, CA 92697-3440, USA \\ \{jwen2,ykow\}@uci.edu, yunanc@ics.uci.edu \\ ${ }^{2}$ The State Key Lab of Software Engineering \\ Wuhan University \\ Wuhan, 430072, China
}

\begin{abstract}
How do online games impact intergenerational family relationships? To answer this question, we investigated QQ Farm, the most popular online game available on a popular Chinese social networking site. We conducted observations and semi-structured interviews with sixteen pairs of Chinese parents and their adult children. Of the sixteen pairs, seven lived locally; nine pairs lived remotely. The findings of this study suggest that online games provide common conversational topics among local family members and enrich family time. Online games with well-designed shared virtual spaces can also help enhance remote families' awareness of each other's real-life activity. For future intergenerational games, we recommend a game design that requires minimal effort, has a 'healthy' theme, and provides features, such as an activity $\log$ and message board, which help maintain game based family communications.
\end{abstract}

Keywords: Social Network Games, Parents-children Relationship, Domestic Play, Generational Gap.

\section{Introduction}

Online games allow hundreds of geographically dispersed players to perform shared activities in the same virtual world [1]. Players come to online gaming sites not only to play, but to socialize [2]. Formerly perceived as merely "games," online game has become a new environment for individuals to mediate social relationships [3][4][5] [6]. While previous studies have focused on how games can strengthen or form new social relationships among players, game play among individuals with existing social relationships, such as intergenerational family members, have seldom been reported in previous literature [3][4][6][7]. In this article, we ask whether online games can be beneficial in reinforcing the social relationships between intergenerational family members with substantial age and value differences.

Intergenerational relationships are of special interest to China, a country where the traditional family structure is rapidly undergoing transformation. The traditional 
Chinese family is a co-residence among three generations of family members. However, co-residence among intergenerational family is becoming increasingly rare as young adults migrate to other regions seeking better employment opportunities [8]. Studies have shown that older parents continue to desire traditional family relations, such as the assistance of their children with housework, being able to care for their grandchildren [11], and keeping mutually close contact with their working children [10]. This raises the question how we can continue to maintain these traditional practices given the modern context where intergenerational relationships are practiced at a distance, rather than in the same household [11].

Given the widespread adoption of online gaming in contemporary Chinese society, we are interested in seeing if online games can serve as a new platform for mediating intergenerational family interactions. We examine QQ Farm, a popular multiplayer online game in China that at its height had 6.8 million users logon simultaneously. QQ Farm has a player pool that includes a large number of older parents and their adult children [12][13]. For our study, we conducted semi-structured interviews to examine how playing QQ Farm impacts the relationships among family members who live locally and those who live remotely. Sixteen pairs of QQ Farm players participated in the study, with a Chinese parent and one adult child in each pair. These pairs include any combination of father or mother with a son or daughter.

The results of the study have shown that social networking games such as QQ Farm can improve the communication among family members locally, and enhance the connectedness between family members remotely. In allowing online players to co-construct a shared social space from which family members can extrapolate into awareness of real world activities, online games can strengthen existing family ties. At the end of this paper, we will introduce opportunities for designing games that support intergenerational play.

\section{Related Work}

Recently, there is an increasing interest in studying social interactions in online game play in the HCI community [4]. Studies have found that social interactions in online games can improve social relationships among players [3] [6] [7], since online games allow large number of players to interact and accomplish shared goals together. Ducheneaut [6] highlights the designing strategy for creating a sense of social presence in online games. Nardi [7] argues that social interactions, particularly those that accomplished shared tasks, are especially desirable within a game environment.

Online game play is increasingly popular among the Chinese. Various reports and news articles often indicate that Chinese parents worry about the negative influence of video games on their children, in particular, the amount of time consumed in game play e.g. [14]. Interestingly, these older adults themselves often engage in and benefit from social gaming activities, such as chess, card, and mahjong [15]. For Chinese parents, social games, when played on computers, often have negative associations, such as game addiction and distraction from school work [14]. Lindtner et.al. [16] [17] found that many urban young Chinese players establish close social ties through game play in Internet cafés [16] and in game-related online communities [17]. These studies show that online gaming has become a meaningful way for youth to get to know friends and help each other in their offline lives. 
While some digital games have been developed for families, these games have restrictive game spaces that only allow a handful of players within the same household. For instance, Age Invaders is an intergenerational game that is designed to bridge family ties by enabling older adults to engage in both in-person and remote play with their children and grandchildren [18]. Others games, such as the Magic Box [19], are developed to encourage the exchange of thought-provoking items between grandparents and grandchildren living remotely, indicating that intergenerational play can be associated with family history, culture, and fun. Voida et al. [20] studied console games and found mutual conversion between the individual-play and family activities during game playing, showing how players might respond differently according to the social relationship they have with other players.

In addition to games, intergenerational relationships can be promoted by other types of digital devices [21] [22]. Digital Family Portrait is designed to transmit iconic projections of older adults' daily activities to their remote family members. Digital Family Portrait allows family members to know whether the older adults are active and well on a daily basis [23]. Similarly, Family Window $(F W)$ is designed as an always-on video media space to connect family members residing in two households through video cameras [24]. Using Family Window, family members can share their daily activities and increase their feelings of connectedness with each other. Devices such as SPARCS [25] and Wayve [26] are also designed to encourage information and photo sharing among family members.

Online games are becoming increasingly popular among the Chinese. Their penetration into intergenerational social space thus providing and the social-cultural landscape continues to transform Chinese families, understanding the possible impact of online gaming in family relationships could be vital. The literature suggests that games and other form of digital devices can be used to leverage intergenerational family interactions; nevertheless, there has not yet a study on the use of online game by intergenerational family members. In what follows, we report the findings of our study on QQ Farm and discuss possible implications for future game design.

\section{The Game - QQ Farm}

QQ Farm is an online game developed by Tencent, Inc. in 2009, and residing in Tencent's social networking site QZone. Social Networking Sites [27] are web-based services that allow users to communicate and construct social connections with other users. And Tencent is the provider of many popular Internet services including the QQ Instant Messenger. On March 31, 2010, it was reported that there were 568.6 million active user accounts on all QQ service platforms including QQ Farm, QZone, and QQ Instant Messenger.

On QQ Farm, players act as farm owners and manage their own farms. Figure 1 shows what QQ Farm looks like in the game environment. In addition to common farming activities such as cultivating, irrigating, and harvesting crops as real farmers do, players can also visit farms owned by their SNS friends. On the farms owned by their friends, players can perform limited actions-some helpful, some mischievous. Helpful actions include weeding and irrigating, whereas mischievous actions include stealing the other players' mature crops. 


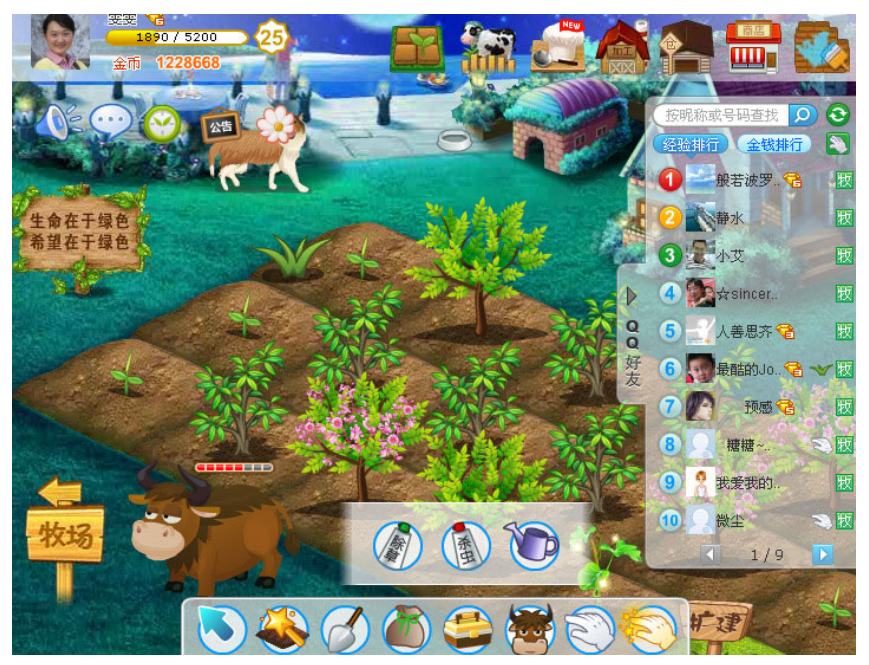

Fig. 1. Screenshot of the first author's QQ Farm

Each activity a player performs on her own farm is rewarded by experience points or, with virtual gold coins through the selling of mature crops. Accumulated experience points can raise the player's level in the game. The higher the player's game level is, the more privileges she has to expand her farming field. Similarly, the more coins the player has, the more "money" she has to purchase expensive crops and fields. Weeding and irrigating for other players are considered helpful in the gaming environment since it helps the other farm owners obtain additional experience points or coins. On the contrary, stealing other players' mature crops is considered a mischievous action since the owner is no longer able to gain virtual gold coins by selling her stolen crops.

In addition to game play itself, each player has access to a personal message board associated with their farm [Figure 2]. The personal message board shows log information about one's farm, including both helpful and mischievous actions. As shown on Figure 2, a player can see who came to visit their farm and when, and what they did to it. Players can also personalize their space through naming and decorating their own farms.

After its launch in 2009, QQ Farm became immediately popular among Chinese players. In general, people were intrigued by the fact that QQ Farm had attracted a large population of older adult players, and the family play of QQ Farm was soon reported by various newspapers and media sources as a new Chinese social phenomenon [12][13]. For instance, Net Ease 163.com, one of major news portal sites in China, published a story about a mother using QQ Farm as an education resource to help her child understand botany [12]. Another article described how a seventy-year old grandma played QQ Farm with her granddaughter remotely [13]. Following these reported stories, we conducted a qualitative study to examine the influence of QQ Farm game play on family relationships. 


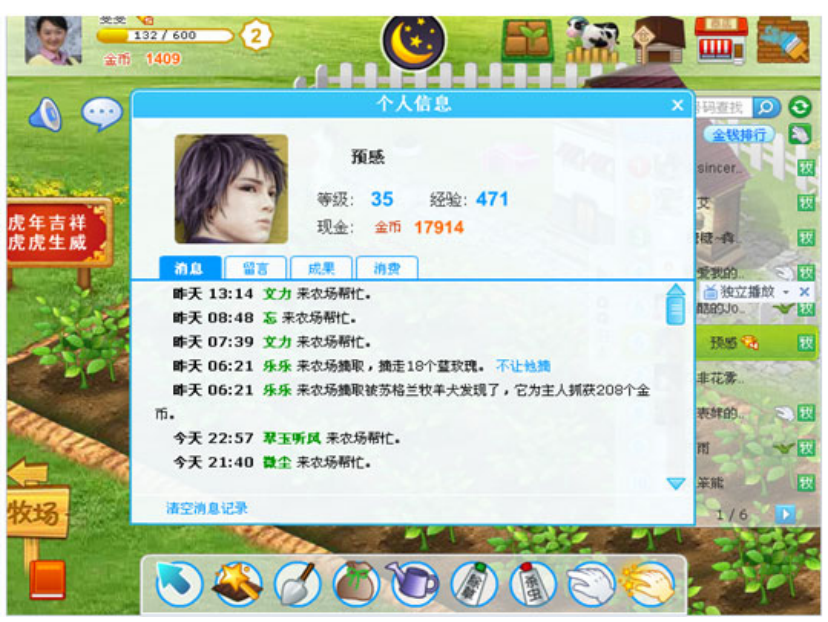

Fig. 2. Screenshot of a personal message board in QQ Farm

\section{Methodology}

We conducted a qualitative study from March 2010 to November 2010. We examine the impact of online gaming on intergenerational family relationships. As described in the literature review section, tensions arise when adult children move out of a home where the parents still hold traditional Chinese family values. We studied only parents and adult children because of this, excluding children below the age of 18, who were more likely to live at home.

\subsection{Participants}

Sixteen pairs of parent-adult children dyads participated in our. Participants were recruited using snowball sampling method. The first two participants were recruited from the university where the authors are affiliated, while the rest of the participants were referred by participants interviewed previously. The snowball sampling method appears to be most appropriate way to recruit family members who play online games due to the social property of QQ Farm.

Of the sixteen parent-adult children dyads we studied, seven pairs live in the same households, or in nearby neighborhoods in the Hubei and Sichuan provinces. We refer to these pairs as "local families" since they were able to meet in person frequently. The other nine pairs lived in different cities. While all the parents we interviewed live in China, their adult children lived remotely in America, Japan, Australia or Denmark. Table 1 summarizes the participants of the study based on their family relationship and local/remote living conditions. The age of parents ranges from 47 to 64 . The age of adult children ranges from 18 to 36 .

These sixteen pairs of participants contain three conditions: both members of the dyad are active players ( 9 pairs); only one member of the dyad (either the parent or the child) is a player (4 pairs); and both members of the dyad are non-players 
Table 1. The parents-children relationships

\begin{tabular}{|l|l|l|l|}
\hline $\begin{array}{l}\text { Parents-children } \\
\text { relationships }\end{array}$ & $\begin{array}{l}\text { Number of } \\
\text { pairs }\end{array}$ & $\begin{array}{l}\text { Local } \\
\text { families }\end{array}$ & $\begin{array}{l}\text { Remote } \\
\text { families }\end{array}$ \\
\hline Father-Daughter & 4 & 2 & 2 \\
\hline Mother-Daughter & 4 & 2 & 2 \\
\hline Father-Son & 3 & 1 & 2 \\
\hline Mother-Son & 5 & 2 & 3 \\
\hline Total & 16 & 7 & 9 \\
\hline
\end{tabular}

(3 pairs). The non-playing participants served as a baseline for us to understand the behaviors of the "dual-play" pairs. By comparing intergenerational communication patterns between "dual-play" pairs with those having one or more non-playing participants, we found that online games could indeed improve intergenerational communications. It is worth mentioning that among the nine pairs of dual-play pairs, four pairs were local and the other five were remote families.

\subsection{Data Collection}

Human subject study approval was obtained from the university where the researchers are affiliated prior to data collection. We acquired verbal consent from every participant and recorded only their screen names used in the games. Nevertheless, all names reported in this paper are pseudonyms.

In the study, we first observed the game play behaviors of the six pairs of dual-play families at QQ Farm for two weeks. Observing game play helped us to understand the basic behaviors participants demonstrated online. We collected the game logs from the personal message board of each participant. These logs recorded all the players' interactions in the game space. The observation data were collected twice a day, once in the morning and once at night. 4132 messages were gathered during the two weeks. Excluding messages relaying the game play records between the farm owner and their other friends, 319 messages were found relating to interactions between family members, accounting for $8 \%$ of the total logged messages.

Based on the family play behaviors observed online, we developed semi-structured interview questions. The interview study was extended to single-play or non-play pairs in order to better understand the impact of game playing on family relationships. The interview questions were centered on game play and family relationships. Specifically, we asked:

-How often do participants play QQ Farm?

-How does participant play with their family members?

-How does the game impact their family relationships?

When possible, we interviewed subjects in-person or by telephone. When in-person or telephone interviews were impossible, we conducted instant messaging interviews. Of the thirty-two subjects, five were interviewed in person, twenty-three were interviewed by telephone, and the remaining four were interviewed through instant 
messaging. The length of the interviews ranged from 30 to 60 minutes. Data were voice recorded transcribed in Chinese, and then translated into English.

\subsection{Data Analysis}

Interview transcriptions were coded using grounded theory [28] to identify recurring themes. The coding process started with the extractions of themes that relate to family relationships. To ensure the dependability of the results, three researchers conducted an independent coding on a sample size respectively, which were four pairs of transcriptions chosen randomly from sixteen pairs of participants. The intergenerational relationships of theses four pairs include a mother and son, father and son, mother and daughter, and father and daughter. After the initial coding, we compared and discussed the four sets of themes coded by each individual researcher. The initial themes and relationships were identified gradually in a manner of convergence.

In the second stage, one researcher coded the rest of the data iteratively based on the initial themes. When all codes and memos were collected, the researchers grouped them in line with the categories or properties they represented. Based on this arrangement of data, this study examines the online game playing behaviors in both local and remote families.

\section{Findings}

In this section, we first describe general game playing behaviors demonstrated through observational data, and then introduce the diverse impact of game play on the local and remote families.

\subsection{Online Game Playing}

As mentioned earlier, we observed six pairs of dual-play families' game activities for two weeks and recorded 319 messages between the six pairs of participants during these two weeks. All families routinely logged onto the game space on a daily basis. Table 2 shows the quantitative data from log analysis. All participants had more than 6 months of gaming experience and reached at least the 15th level according to the game ranks designed in QQ Farm (0 as the lowest and 50 as the highest in the current game design). The participants logged on 1 to 6 times per day, with 5 to 30 minutes spent on the game space each time. The log analysis indicates that the participants played QQ Farm in a relatively flexible manner as the game does not require players to play simultaneously over a long period of time.

Some of our participants played QQ Farm while engaging in their other daily activities. Figure 3 shows a participant studying while playing QQ Farm. This participant told us that he was afraid his mature crops might be stolen if he did not gather them in time. He took occasional glances at his farm while studying. Because QQ Farm does not require undivided attention, and allows players to perform other tasks while playing the game, most of our participants had considered the game as less intrusive than most other video games. Thus, our participants were more receptive to QQ Farm than other video games. 
Table 2. Log analysis of six pairs of dual-play families

\begin{tabular}{|c|c|c|c|c|c|c|}
\hline $\begin{array}{l}\text { Relation } \\
\text { pairs }\end{array}$ & $\begin{array}{l}\text { Parents- } \\
\text { children } \\
\text { relationship }\end{array}$ & Age & $\begin{array}{l}\text { Game ranks } \\
\text { (Levels) }\end{array}$ & $\begin{array}{l}\text { Logins } \\
\text { (per day) }\end{array}$ & $\begin{array}{l}\text { Playing } \\
\text { Time } \\
\text { (Minutes } \\
\text { per day) }\end{array}$ & $\begin{array}{l}\text { Interaction } \\
\text { messages } \\
(2 \text { weeks) }\end{array}$ \\
\hline \multirow[t]{2}{*}{ R1 } & Father & 54 & 38 & 6 & 30 & \multirow[t]{2}{*}{93} \\
\hline & Daughter & 28 & 18 & 6 & 25 & \\
\hline \multirow[t]{2}{*}{$\mathrm{R} 2$} & Father & 63 & 19 & 2 & 5 & \multirow[t]{2}{*}{58} \\
\hline & Son & 28 & 15 & 5 & 20 & \\
\hline \multirow[t]{2}{*}{ R3 } & Mother & 63 & 22 & 3 & 10 & \multirow[t]{2}{*}{47} \\
\hline & Son & 28 & 15 & 5 & 20 & \\
\hline \multirow[t]{2}{*}{$\mathrm{R} 4$} & Father & 63 & 34 & 1 & 5 & \multirow[t]{2}{*}{37} \\
\hline & Daughter & 34 & 19 & 5 & 15 & \\
\hline \multirow[t]{2}{*}{ R5 } & Mother & 60 & 41 & 6 & 30 & \multirow[t]{2}{*}{49} \\
\hline & Son & 29 & 39 & 4 & 15 & \\
\hline \multirow[t]{2}{*}{ R6 } & Mother & 65 & 26 & 6 & 30 & \multirow[t]{2}{*}{35} \\
\hline & Daughter & 36 & 24 & 5 & 10 & \\
\hline
\end{tabular}

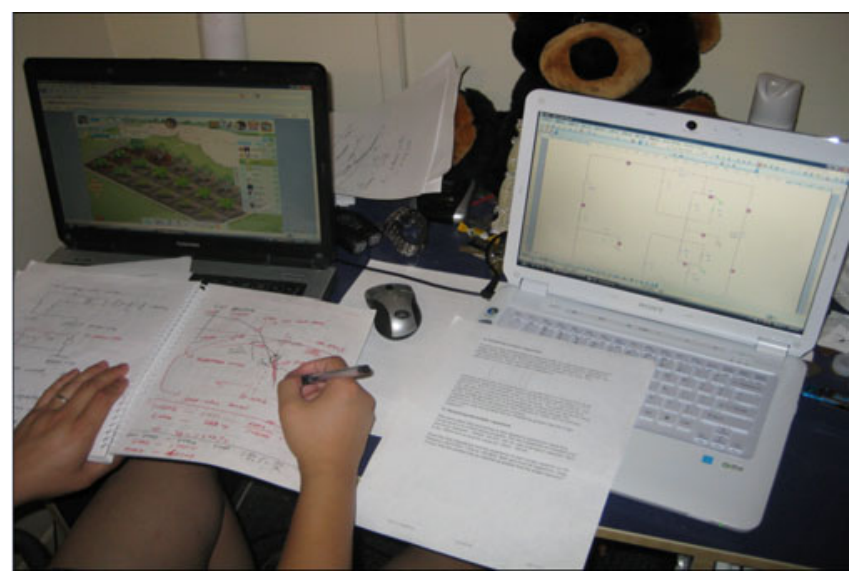

Fig. 3. Playing QQ Farm but not delaying the studying

Our study suggests that Chinese parents and their adult children had different motivations when playing online games. Most parents started playing because their children were playing, whereas the adult children played because they were interested in the game itself. As one participant said:

It was amazing to watch the growth process of different plants...

This game let me know what some regional plant species look like and how they grow.

Farming in the game space provides players with an opportunity to connect with a completely new environment and helps them relax away from their daily routines. The game space also serves as a social space in which family can interact. As a 
game-based interaction, QQ Farm greatly strengthens social ties and family relationships. The dual-play families frequently interacted in the game space, regardless of whether they resided at the same household or not. Family members visited each other's farm from 3 to 24 times a day. (Note: each game log in may be associated with multiple visits). The dual-play dyads admitted that they like to check on each other's farms; they liked to know what plants their parents or children were cultivating and whether they could steal or help each other in the game space. The other forms of interactions among family members, such as face-to-face talks, phone calls, or emails may only happen once or twice a week on average; but dual-play parents and children interacted on the game space multiple times a day. Noteworthy is that although we collected data on all types of family play behaviors, the comparison among dual-play, single-play and non-play families is not the main focus of the current paper; rather, the non-play group served as a basis from which we could understand dual-play behaviors.

\subsection{Online Game Adding Joy to Conversations among Local Families}

The local families live in the same household or in nearby neighborhoods and can see or visit each other frequently. However, the close physical proximity did not always correspondent to tight family relationships. Differences in education, culture, and values between the dyads often lead to conflict and tension in their relationships [9]. Counter intuitively, this tension may be amplified among local families due to the frequent face-to-face interactions.

In local family dyads, QQ Farm mediated interactions between family members such that their relationship may improve. For example, Su is the primary caregiver for her elderly father and lives in the same household with him. Their interactions were often intense due to frequent disagreement in care-giving activities. They found a way to show concern to each other through QQ Farm.

Su: daughter, 36 years old, living in Chongqing city:

This game [QQ Farm] opened a door to us to become farmers--the role we couldn't be in the practical world, it was exciting that we can plant and harvest just like a real farmer...We found more things to talk about and found more joy in our conversation... when I found that my father's crops were mature, I would remind him to gather them. If I were at home, I would tell him in-person, else, I would give him a call...

In Chinese, “joy愉快” means happiness 欢乐, and lightheartedness 轻松. This example shows how online game like QQ Farm provides new ways which pleasant conversation can happen among family members.

At the same time, when daily conversation becomes intense, gaming activities can act as pretexts and reasons for making phone calls to family members. For local families, the barriers in maintaining close family ties are not due to the lack of faceto-face interactions, but rather due to differences in values between the two generations. In this situation, game space allows both parties to engage in conversations where these disagreements need not arise. 
Even for local families that have well-maintained family ties, QQ Farm can enrich shared family time. Wang is a retired mother with two adult daughters. In Wang's experience, QQ Farm reinforces their existing family ties.

Wang, 63 years old, living in Wuhan city:

Our family members were all playing QQ Farm... every time my daughters and their families came visit, we shared tips on how to play the game...my grandchild would sometimes join us [at her computer]to play together...this game fascinated everyone in our family!

In Wang's case, her children and grandchild played with her and shared the experiences that occurred in the game space in real-life interactions. The use of a shared computer contributes to the building of family ties since family members have reasons to occupy the same room and talk. Here the QQ Farm game was integrated into family time, and turned into a new family activity.

\subsection{Online Game Serves as an Awareness System for Remote Family Members}

Unlike local families, remote families are not able to see each other on a daily basis. Family members may also live in different time zones making it harder to make phone calls. In this case, family interactions may rely on either the common leisure time they have or on asynchronous communication that happens infrequently. Resonating with this study [29], non-playing family groups living at long-distances from each other also reported the challenge of staying connected and maintaining close family ties with each other.

Compared with the non-play families, dual-play families use the shared game spaces as another way to interact with family members. In other words, game play is turned into a family interaction that ties family members closer together. Zhang is a mother who was proud that both her son and daughter were attending graduate schools in the United States. Due to the time differences between the US and China As a result, Zhang could not make as many phone calls with her children as she had liked. When Zhang had free time, her son and daughter were studying in the schools; and when her children were free, Zhang was working at her company. Despite these barriers, Zhang managed to connect with her children daily through QQ Farm.

\section{Zhang: mother, 57 years old, living in Wuhan city:}

Last year, my son and daughter went to the US. I missed them very much... Whenever we met in the farms, I feel that they were just next to me... At times I would be too busy to manage my farm and my children would help me in the game space, and that makes me happy.

Parents like Zhang find social connections with their children when their children visit their farms. The farms, as shared virtual spaces, allow players to perform activities that greatly affect each other beyond the game space. Zhang perceives the helpful actions in the game to be representative of how much her children care for her. In this case, family ties are strengthened through seemingly trivial game activities. Because of its asynchronous nature, this game play is able to enrich otherwise intermittent family interactions, when these other forms of interactions become more difficult. 
The game space gives remote family members the opportunity to observe each other's online activities. And interestingly, this online observation had allowed many participants stay cognizant of their family members' activities in real-life. Jean, a Ph.D. student of computer science told us how she would know where her father was.

Jean, daughter, 28 years old, living in California:
I love to guess at what my father is doing by examining his activity log...for instance, when he went on business trips, his farm would become unattended for a while... If later I found his farm gets cleaned up, crops gathered, and new crops sown, I know that he had come back home...

For Jean, the game space allows her to guess father's real-life daily activities based on his game activities. In this sense, the use of online gaming is turned into a system through which players are able to stay aware of each other's daily life [21]. Like Digital Family Portrait [23], our study suggests that the game space also has the potential to deliver awareness information amongst family members, although in a less direct manner. In the game space, a person can identify another player's daily game routines to discover atypical behaviors that may be influenced by an event that has happened in real-life. Our participants had not found such observations to be invasive of their private life. But rather, they were alternate ways for family members to care for each other.

Some studies have found that working children have treated communication devices such as the Digital Family Portrait as a replacement for the occasional phone call [23]. However, we have not found this to be a problem for online games such as QQ Farm. The way of interacting with family members can also be used purposely to deliver certain information. Yong, an elderly mother living in the countryside, created a personalized message on her farm to show how she missed her son.

Yong, mother, 64 years old, living in Hubei province:

I believed this farm can convey emotional feelings... I changed the name of my farm to “come home whenever you can”看常回家看看. I hoped my son would come home as often as he can. I know that he is busy [with school work] so I do not want to tell him too directly [or he may get distracted].

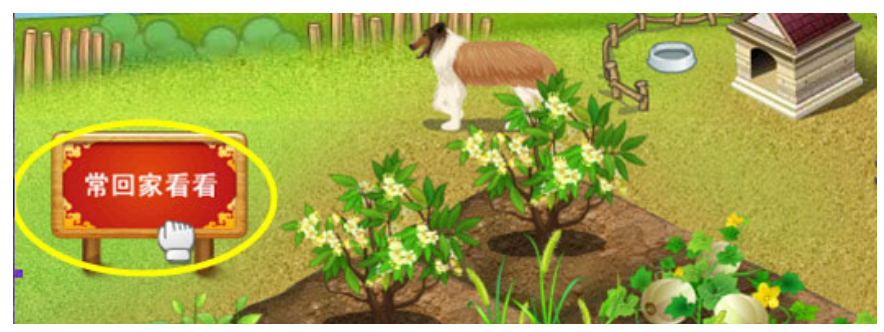

Fig. 4. The bulletin board in Yong's field 
As shown in Figure 4, Yong's personalized message indicated that she had wished that her son would visit her more frequently. However, Yong had also wanted to avoid bringing up her feelings in telephone calls for it may become an emotional burden for her distant son. As a result, she used QQ Farm as a system to deliver this message subtly. In this case, the game space was used to deliver messages that may be too sensitive to bring up in direct conversation.

As described in this section, online gaming can connect distant family members. In addition, the game space also allows family members to stay cognizant of each other's real-life activities. QQ Farm can also be used to convey information that is inappropriate in direct conversation.

\section{Discussion}

In summary, our research suggests that online games can bolster intergenerational relationships. Online gaming can support intergenerational relationships in two primary ways. First, it generates new conversational topics for local families. Second, it can be used to track the real-life activities of family living at a distance. In this section, we discuss how online games can reinforce existing family relationships.

Online games become common conversational topics for both parents and adult children. When game activities become a family conversation topic, the tension caused by generation gaps is hidden beneath the engagement of game play. When family members live apart, common conversation topics may be difficult to establish between intergenerational family members, since they may have very few common daily activities to share. Discussing online games often ignites fruitful conversations that eventually strengthen intergenerational relationships.

The second finding is that online games can act as an awareness system to keep family members informed about each other's daily activities, since real-life daily routines are often reflected in online activity patterns, e.g., if Jean's father changes a real-life activity, his online activity is likely to be affected as well. As we saw in Yong's story, online activity can also be manipulated to express unspoken needs or concerns to family members.

Furthermore, online games provide awareness information for family members that are geographically distributed. It is critical for family members to co-construct a shared virtual space that keeps logging activities that can be seen by each other. A shared virtual space provides opportunity for players to leave traces of actions that are associated with their real-life activities. Online traces, such as when and what the other family member was engaged in, can be extrapolated into an awareness system of a player's real-life activity.

The first finding suggests that the importance of designing a game space that is acceptable by both generations. Games with themes that are perceived by older parents as "healthy" are more likely to be adopted as a family play platform. QQ Farm is considered a healthy and educational environment since parents believe their children can learn agriculture and botany knowledge through game play. The playful activities embedded in the game arouse participants' interests in the game and sustain their interpersonal interactions that are mediated through the game play. 
In QQ Farm, two features allow players to trace game activities online: the activity $\log$ and the message board. The activity log automatically records activities associated with a certain farm; and the message board can be used as a proactive communication tool for players to pass on information to other players intentionally. Our findings suggest that both features can help family members become more aware of each other's activities.

For family members living apart, the game keeps them aware of some of their remote members' activities, but also augments and enriches existing communications, such as increasing the chances of telephone conversations as well the conservation topics. Beyond family members, closely connected players in other social relationships can also extrapolate each other's real-life activities from their virtual activity patterns, suggesting that online games may similarly augment other real-life relationships when social and cultural barriers hinder other forms of communications, such as in some corporate workgroups.

\section{Conclusion}

The paper highlights how online games can support intergenerational relationships. Online games can serve to strengthen social bonds underneath the pretense of online game play. Online games can also serve as an awareness system to help derive remote family member's real-life activities. But to achieve these benefits, designers need to develop a game that appeals to players of widely varied ages and backgrounds. Some of the design considerations that make a game truly intergenerational include: requiring minimal effort yet scalable to other committed players, containing themes that appeal to user of all ages, and providing features such as activity log and message board that capture online activities and communication.

\section{References}

1. Flew, T., Humphreys, S.: Games: Technology, Industry, Culture, New Media: an Introduction, 2nd edn., pp. 101-114. Oxford University Press, South Melbourne (2005)

2. Castells, M.: The Internet Galaxy, pp. 9-35. Oxford University Press, Oxford (2001)

3. Nardi, B., Harris, J.: Strangers and friends: collaborative play in world of warcraft. In: Proc.CSCW 2006 (November 4-8, 2006)

4. Williams, D., Ducheneaut, N., Xiong, L., Zhang, Y., Yee, N., Nickell, E.: From Tree House to Barracks: The Social Life of Guilds in World of Warcraft, Games and Culture, vol. 1(4) (October 2006)

5. Voida, A., Greenberg, S.: Wii all play: The console game as a computational meeting place. In: Proceedings of the ACM SIGCHI Conference on Human Factors in Computing Systems (CHI 2009), Boston, Massachusetts, April 4-9, pp. 1559-1568. ACM Press, New York (2009)

6. Ducheneaut, N., Yee, N., Nickell, E., Moore, R.J.: "Alone together?" Exploring the social dynamics of massively multiplayer online games. In: CHI 2006: Proceedings of the ACM Conference on Human-Factors in Computing Systems. ACM Press, NY (2006)

7. Nardi, B.: My Life as a Night Elf Priest: An Anthropological Account of World of Warcraft. Digital Culture Books, Ann Arbor (2010) 
8. Sheng, X., Settles, B.H.: Intergenerational Relationships and Elderly Care in China: A Global Perspective. Current Sociology 54, 293 (2006)

9. Goh, E.C.L., Kuczynski, L.: 'Only children' and their coalition of parents: Considering grandparents and parents as joint caregivers in urban Xiamen. Asian Journal of Social Psychology 13, 221-231 (2010)

10. Chen, X., Silverstein, M.: Intergenerational Social Support and the Psychological WellBeing of Older Parents in China. Research on Aging 22(1), 43-65 (2000)

11. Silverstein, M., Cong, Z., Li, S.: Intergenerational Transfers and Living Arrangements of Older People in Rural China: Consequences for Psychological Well-Being. J. Gerontol B Psychol. Sci. Soc. Sci. 61(5), S256-S266 (2006)

12. The report of QQ Farm from Chinese website NetEase (163.com), http://tech.163.com/10/0730/11/6CR9G68A00094IL6.html (retrieved September 18, 2010)

13. The report of QQ Farm from Chinese website duowan.com (retrieved September 18, 2010), http: / / web. duowan.com/1004/136290607498.html

14. Alex, G., Kate, L.: Just Like the Qing Empire. Internet Addiction, MMOGs, and Moral Crisis in Contemporary Chi, Games and Culture 3(1), 59-75 (2008)

15. Glei, D., Landau, D., Goldman, N., Chuang, Y.L., Rodriguez, G., Weinstein, M.: Participating in social activities helps preserve cognitive function: an analysis of a longitudinal, population-based study of the elderly. Int. J. Epidemiol 34, 864-871 (2005)

16. Lindtner, S., Mainwaring, S., Dourish, P., Wang, Y.: Situating Productive Play: Online Gaming Practices and Guanxi in China. In: Proceedings of the 12th IFIP TC 13 International Conference on Human-Computer Interaction: Part I, pp. 328-341 (2009)

17. Kow, Y.M., Nardi, B.: Culture and Creativity: World of Warcraft Modding in China and the U.S. In: Bainbridge (ed.) Online Worlds: Convergence of the Real and the Virtual. Springer, London (2009)

18. Khoo, E.T., Lee, S.P., Cheok, A.D., Kodagoda, S., Zhou, Y., Toh, G.S.: Age Invaders: social and physical intergenerational family entertainment. In: Proc.CHI 2006, pp. 243-247. ACM Press, New York (2006)

19. Hilary, D., Frank, V., Peter, F., Martin, G., Steve, H.: "I Wish We Could Get Together": Exploring Intergenerational Play across a Distance via a 'Magic Box'. Journal of Intergenerational Relationships (2008)

20. Voida, A., Carpendale, S., Greenberg, S.: The Individual and the Group in Console Gaming. In: Proceedings of the 2010 ACM Conference on Computer Supported Cooperative Work, Savannah, Georgia, USA, pp. 371-380 (2010)

21. Romero, N., Markopoulos, P., Baren, J., Ruyter, B., Jsselsteijn, W., Farshchian, B.: Connecting the Family with Awareness Systems, Personal and Ubiquitous Computing, vol. 11. Springer, Heidelberg (2006)

22. Tee, K., Brush, A.J.B., Inkpen, K.M.: Exploring communication and sharing between extended families. International Journal of Human-Computer Studies 67(2), 128-138 (2009)

23. Rowan, J., Mynatt, E.D.: Digital Family Portrait Field Trial: Support for Aging in Place. In: Proc. CHI 2005, pp. 521-530. ACM Press, New York (2005)

24. Tejinder, K.J., Carman, T.K., Neustaedter, C., Andrew, F., Kurtz, A.F.: The Family Window: The Design and Evaluation of a Domestic Media Space. In: Proc. CHI 2010: We Are Family, pp. 2361-2370. ACM Press, Atlanta (2010)

25. Brush, A.J., Inkpen, k., Tee, k.: SPARCS: exploring sharing suggestions to enhance family connectedness. In: Proc. CSCW 2008, Savannah, Georgia, USA, pp. 629-638 (2008) 
26. Lindley, S.E., Harper, R.H.R., Sellen, A.: Designing a Technological Playground: A Field Study of the Emergence of Play in Household Messaging. In: Proc.CHI 2010: We Are Family, Atlanta, GA, USA (April 10-15, 2010)

27. Boyd, D., Ellison, N.: Social Network Sites: Definition, History, and Scholarship. Journal of Computer-Mediated Communication 13(1) (2007)

28. Glaser, B.G., Strauss, A.L.: The discovery of grounded theory: Strategies for qualitative research. Aldine de Gruyter., Hawthorne, NY (1967)

29. Cao, X., Abigail, S., Brush, A.J., David, K., Darren, E., Ding, X.: Understanding Family Communication across Time Zone. In: Proc. CSCW 2008, Savannah, Georgia, USA, pp. $155-158$ (2008) 